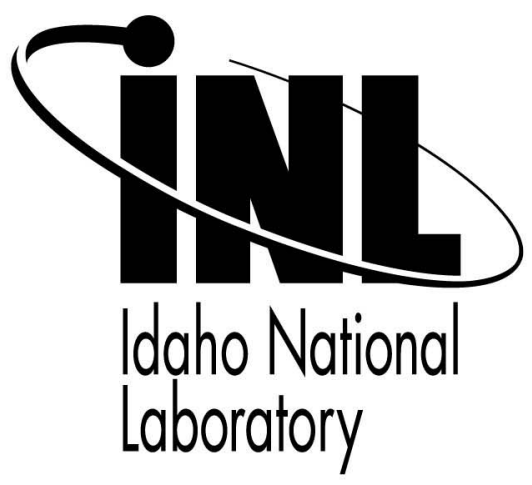

\section{Mental Representations Formed From Educational Website Formats}

\section{Human Factors \& Ergonomics Society}

\author{
Elizabeth T. Cady \\ Kimberly R. Raddatz \\ Tuan Q. Tran \\ Bernardo de la Garza \\ Peter D. Elgin
}

\section{October 2006}

This is a preprint of a paper intended for publication in a journal or proceedings. Since changes may be made before publication, this preprint should not be cited or reproduced without permission of the author. This document was prepared as an account of work sponsored by an agency of the United States Government. Neither the United States Government nor any agency thereof, or any of their employees, makes any warranty, expressed or implied, or assumes any legal liability or responsibility for any third party's use, or the results of such use, of any information, apparatus, product or process disclosed in this report, or represents that its use by such third party would not infringe privately owned rights. The views expressed in this paper are not necessarily those of the United States Government or the sponsoring agency. 


\title{
Mental Representations Formed From Educational Website Formats
}

\author{
Elizabeth T. Cady ${ }^{1}$, Kimberly R. Raddatz ${ }^{1}$, Tuan Q. Tran ${ }^{1,2}$, Bernardo de la Garza ${ }^{1}$, and Peter D. Elgin ${ }^{1}$ \\ ${ }^{1}$ Kansas State University, Manhattan, KS \\ and \\ ${ }^{2}$ Idaho National Laboratory, Idaho Falls, ID
}

\begin{abstract}
The increasing popularity of web-based distance education places high demand on distance educators to format web pages to facilitate learning. However, limited guidelines exist regarding appropriate writing styles for web-based distance education. This study investigated the effect of four different writing styles on reader's mental representation of hypertext. Participants studied hypertext written in one of four web-writing styles (e.g., concise, scannable, objective, and combined) and were then administered a cued association task intended to measure their mental representations of the hypertext. It is hypothesized that the scannable and combined styles will bias readers to scan rather than elaborately read, which may result in less dense mental representations (as identified through Pathfinder analysis) relative to the objective and concise writing styles. Further, the use of more descriptors in the objective writing style will lead to better integration of ideas and more dense mental representations than the concise writing style.
\end{abstract}

While advanced technologies allow educators to present classroom material on the Internet, little guidance exists on formatting distance education materials to facilitate learning. Although there are principles for global presentation of learning content (e.g. SCORM; ADL, 2004), educators choose how to present information for distance learning. Past research suggests that webwriting styles influence the amount of information the reader remembers. Specifically, Morkes and Nielson (1997) identified four web-writing styles that influence memory for website content.

The four writing styles vary in structure (bulleted list or paragraph) and presence of extraneous information. The objective style closely resembles traditional printed educational text, with full paragraphs and many superfluous descriptive words. The scannable style contains superfluous wording formatted in bulleted lists. Concise writing omits superfluous text in paragraph form, while the combined writing style omits descriptors in bulleted lists. These writing styles are presented in Figure 1. Previous studies used traditional testing measures such as free recall, guided recall, and multiplechoice questions to assess the effect of writing style on memory. Morkes and Nielsen (1997) found improved memory scores for participants who read concise or scannable text, compared with the objective style. Other studies, however, failed to find any differences attributed to web-writing styles within distance education contexts (e.g., Jones, Elgin, Johnson, \& Farris, 2004). These mixed results suggest that writing style may not be as influential on memory for hypertext as originally thought.

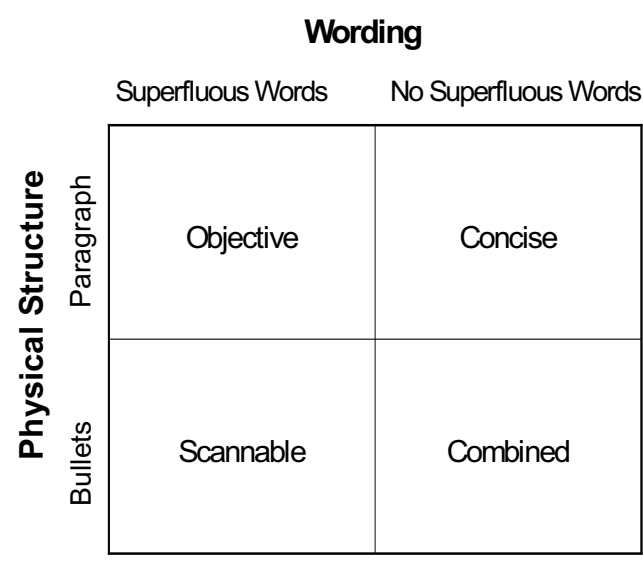

Figure 1. Illustration of the four writing styles used for web-based content.

However, traditional testing metrics may solely assess rote memory ability rather than learning. Although interwoven, memory can be conceptualized as accurate retrieval of prior information whereas learning can be conceptualized not only as accurate retrieval of prior information, but also as updating old information with new information and extending new information to novel situations (Kintsch, 1998). 
Thus, perhaps learning differed as a function of writing style; however, the testing measures used (e.g., multiple choice) may have compelled participants to rely primarily on a particular level of text representation that did not differ across writing styles. Hence, the null findings from previous research may have been due to measurement insensitivity.

\section{Applying Construction Integration Theory}

According to Kintsch's (1998) ConstructionIntegration (CI) theory, readers comprehend content at three levels of text representation within two major phases: a construction phase and an integration phase (see Figure 2). During the construction phase, three text representation levels (surface level, textbase level and a situation model) work in conjunction in a strictly bottom-up manner.

The surface level is the minimally processed text information that is initially encoded. For example, given "One creature in the forest is the nightstalker," surface level processing reveals the sentence's syntactic and orthographic structure, although it is short-lived.

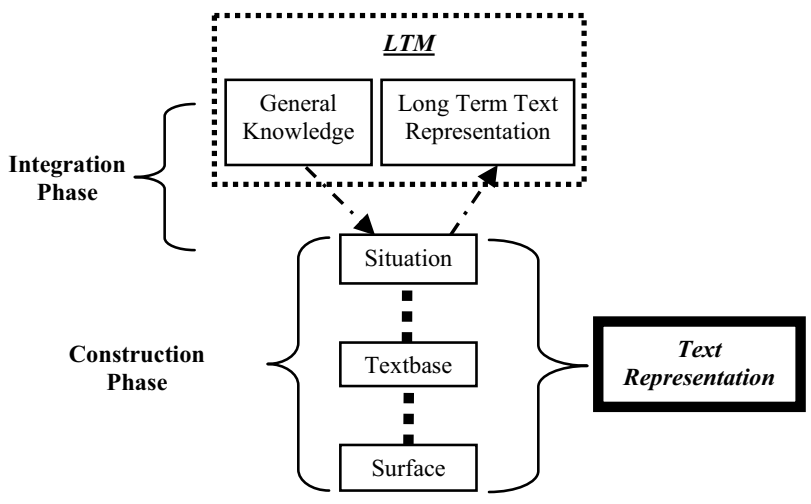

Figure 2. Simplified diagram of the ConstructionIntegration Theory.

At the textbase level, information from the surface level is propositionalized into a predicate (expressed in the surface level as verbs, adjectives, or adverbs) and argument(s) that are mentioned explicitly in the text with minimum inferences. Propositionalizing the above example:

$$
\mathrm{P}_{1}=i s \text { [night stalker, one creature] }
$$$$
\mathrm{P}_{2}=\text { in [forest, night stalker]. }
$$

At the situation model, the textbase level activates relevant and irrelevant information in long-term memory (LTM) to generate elaborative inferences beyond what is explicitly stated. For example, upon reading "One creature in the forest is the nightstalker", the reader may activate concepts such as "bat" or other forest animals as well as activating concepts associated with a nightstalker, such as a "serial killer", "terror", etc. Finally, the integration phase uses general topic knowledge in LTM to refine the situation model to be more context-dependent, leading to heightened activation of context-relevant concepts (e.g., "terror") and deactivation of context-irrelevant concepts (e.g., "serial killer").

The CI model postulates that readers focus attention on constructing a mental text representation at any one level, leading to stronger representation at that level. For example, proofreading involves attention to the surface level, whereas reading for meaning requires attention to the textbase level. When elaborating and connecting current text with prior knowledge to draw elaborate inferences that go beyond explicit text meaning, attention is largely focused on the situation model. Learning relies heavily upon situation models, and the goals of distance education are to help students remember and learn information; thus materials should be formatted to support the development and revisal of the situation model.

Thus, with respect to CI theory, the null results found in past research (e.g., Jones et al., 2004) may have been the result of traditional testing metrics tapping into readers' textbase level, where readers' text representations may be similar across different writing styles. Therefore, this study examined whether the writing styles support construction of similar textbase representations but different situation models. Further, this study investigated whether differences in situation models correlate with better performance on measures that require creativity and the elaboration of inferences.

\section{METHOD}

\section{Participants}

Seventy-two undergraduates participated for course credit. 


\section{Experimental Design}

A between-subjects design was implemented with writing style as the independent variable. Participants were randomly assigned to conditions and tested individually. A cued association task (see below) was used to elicit participants' situation models. The primary dependent variables were the number and order of recalled associates in the cued association task.

\section{Materials}

Participants read the contents of an 11-page website describing a fictional planet "Cyrus" and its fictional wildlife (Shapiro, 1999). The Cyrus website was structured hierarchically and consisted of a home page, two wildlife categories, and four animals under each wildlife category (Figure 3). The website information was novel, thus ensuring that participants had similar amounts and types of previous knowledge and experiences available to facilitate learning. Note that several animals described were similar to common Earth animals, allowing for some related domain knowledge to affect learning.

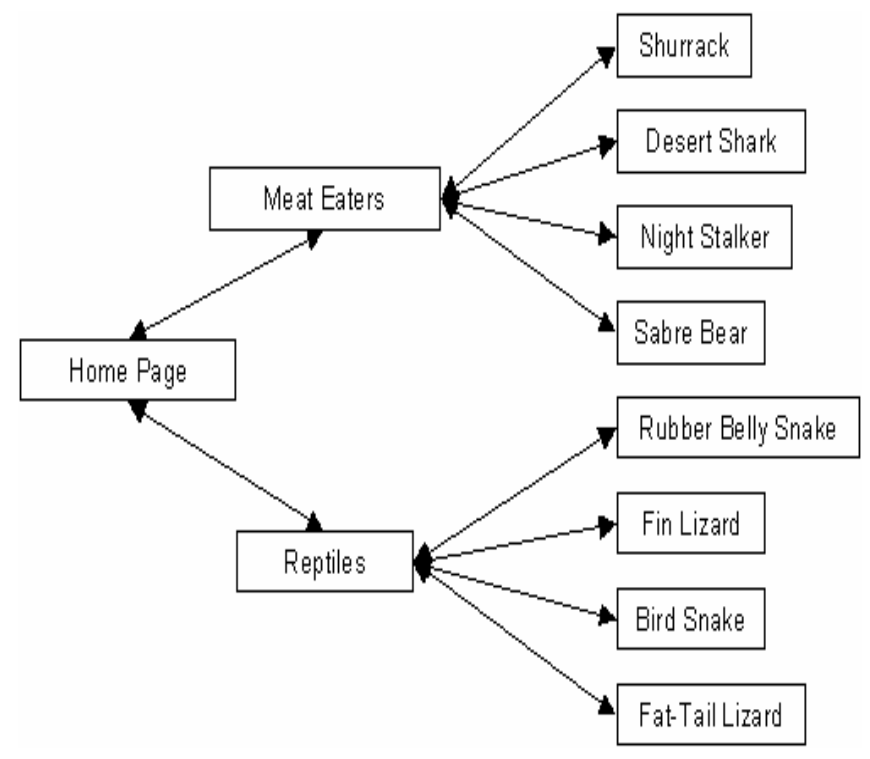

Figure 3. Website hierarchy for Cyrus.

The information was formatted into websites based on each of the four different writing styles depicted in Figure 4. In the objective writing style, information was written in a strictly factual manner typical for distance education material. The concise writing style was similar to the objective condition except superfluous information (e.g., descriptive adjectives) was omitted. Information in the scannable writing style was formatted to support scanning rather than reading word-for-word by employing bullets and incomplete sentences with bolded key phrases and terms. Lastly, the combined writing style was an aggregate of the concise and scannable styles.

A cued association task was used to elicit participants' situation models (Ferstl \& Kintsch, 1999). Participants were presented with a list of cues (presented individually and randomly) that reflected important passage ideas. Participants were asked to produce as many ideas or associates closely related to that cue as they could in one minute. Participants were not given any guidance as to how the concepts should be related and were told there were no incorrect answers and that they should write down all the concepts that came to mind.

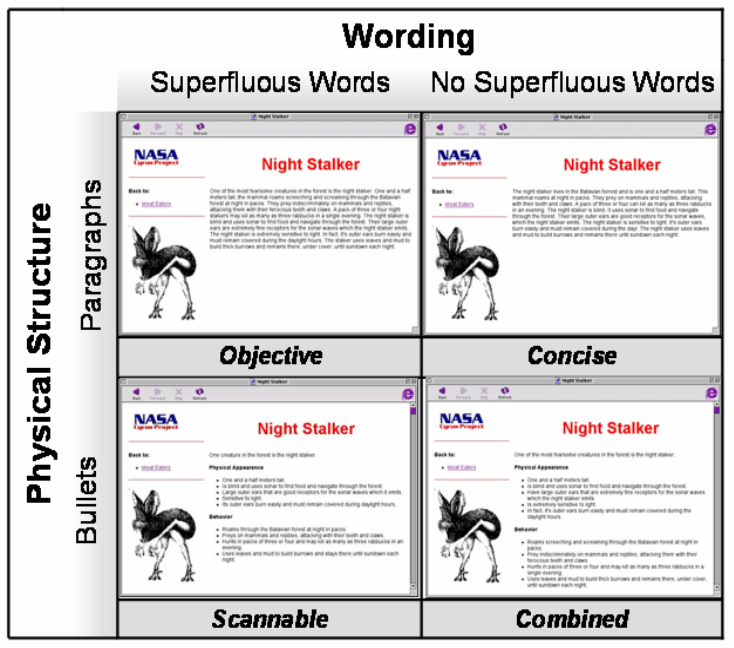

Figure 4. Depiction of how manipulations in physical structure and wording combined to form the four web-writing styles and corresponding illustrations of how website information appeared when it was formatted according to each style.

\section{Procedure}

After familiarization with browser navigation, participants started at the homepage and were instructed to study the Cyrus material in 
preparation for an exam. Participants were not allowed to take notes. Consistent with the study time allotted by Jones et al. (2004), participants were allowed a maximum of 25 min to study the website, although many participants elected to end studying prematurely. Following the study session, participants completed the cued association task, the deep comprehension task, and a demographics questionnaire. The deep comprehension test required participants to complete five essay questions whose answers were not explicitly stated in the text (and therefore required inferences).

\section{Analyses}

Cued association scores. The order in which the participants produced the associates reflects the degree of relationship or association between those ideas and the cue. To score these relationships, the first two ideas produced (i.e., ideas 1-2) were assigned a weight of 1 , the next two ideas (i.e., ideas 3-4) a weight of $1 / 2$, and the last two ideas (i.e., ideas 5-6) a weight of $1 / 3$ (Ferstl \& Kintsch, 1999). To assess the influence of writing style on the cued association task, each passage was propositionalized (i.e., parsed into its meaningful units) independently by two raters to determine argument overlap between ideas in the text. This resulted in the "gold standard" situation model. A score was obtained for each participant by summing their recalled ideas that were actually in the text passages and dividing by the sum of all possible associations in the text passages. Examining the quantity of association of ideas in the actual text provides an index to how well each writing style promotes associative learning (i.e. integration of textual information).

Pathfinder Analysis. In addition to quantitatively analyzing cued association scores, a more qualitative content-based differentiation of the situation models will be performed using Pathfinder network scaling on the weights already assigned to the generated associates. Pathfinder is a structural modeling technique developed to derive and represent the organization of information in LTM through the production of link-weighted networks in which concepts are depicted as nodes and relationships are depicted by links connecting the nodes (Schvaneveldt, 1990).
Deep comprehension scores. Answers given by participants to each of the five essay questions comprising the deep comprehension test were parsed independently into idea units by two raters. Each idea unit was also independently classified as either originating from the text or from an inference. A text score and an inference score were calculated for each participant.

\section{PREDICTED RESULTS}

Based on CI theory, which suggests that all four writing styles have similar textbase levels of representation, but do not promote similar situation models, it is hypothesized that formats facilitating scanning may contribute to less developed situation models because the text is scanned instead of read word-for-word. Readers who scan may fail to attend to contextual information important for developing rich situation models. Conversely, it is hypothesized that traditional paragraph styles will produce better situation models because readers are more likely to read word-for-word, thereby facilitating comprehension and developing strong situation models. Thus, it is hypothesized that scannable and combined styles will produce poorly constructed situation models relative to objective and concise writing. Insofar as lower cue association scores indicate less accurate situation models, the scannable and combined writing will have lower cue association scores compared to objective and concise writing (Figure 4).

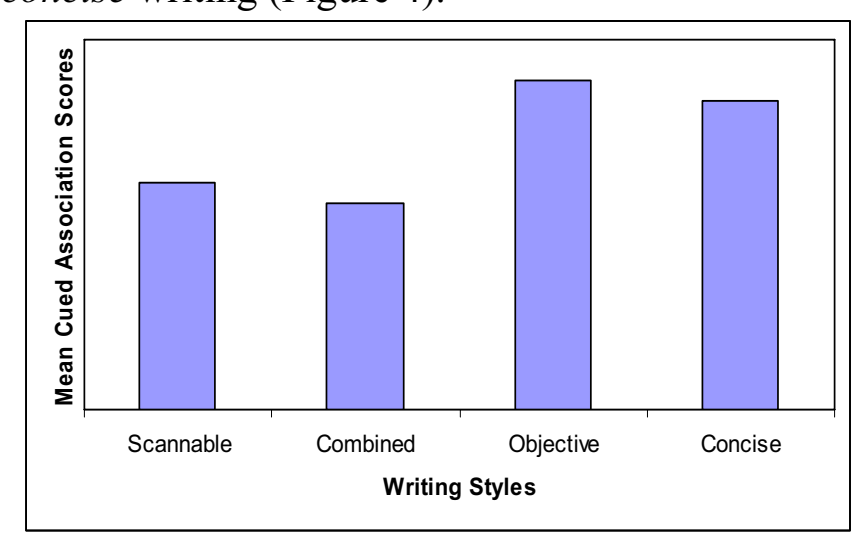

Figure 4. Extent to which participants' situation models are predicted to develop as a function of writing style (based on cued association scores). 
Further, Pathfinder analysis allows for measuring similarities among networks. Thus, the overall similarity between situation models developed from each writing style and between the situation models and the "gold standard" situation model will be assessed. From arguments above, it is hypothesized that situation models resulting from the objective and concise writing styles will be similar to each other and to the "gold standard" situation model and will also contain more associations based on general knowledge that were not reinforced in the text but were plausible, based on the context (e.g., nightstalker - bat-like; nightstalker - terrifying). Conversely, situation models from scannable and combined writing styles will largely contain associations made within the text (e.g., nightstalker - forest). Lastly, it is predicted that participants with situation models highly consistent with the "gold standard" will make significantly more inferences on the deep comprehension test.

\section{GENERAL DISCUSSION}

Both the physical structure and the wording of hypertext can impact learning through distance education. Text formatted with bullets, bolded key sentences, shorter text sections, etc., may help readers organize information but at the cost of inducing scanning behavior which may lead to shallow text processing. Conversely, text in paragraph form may force readers to read the text word-for-word (as opposed to scanning), thereby processing information at a deeper level.

Hypertext wording may also affect memory and learning. For example, superfluous adjectives (e.g. "fearsome," "extremely," "ferocious") may help capture the reader's interest and compel her to immerse herself into the fictional world and create imaginary experiences with the creatures described (Zwaan, 2004). Alternatively, superfluous words may distract from important information, thereby adversely affecting memory (Mayers, 2001).

Materials used in distance education courses must be able to facilitate learning while compensating for challenges imposed by the asynchronous teaching environments. This study adopted a text comprehension framework to investigate how web-writing styles influence readers' abilities to associate concepts within the text to concepts stored in LTM. Results should provide an initial understanding of how text information is acquired and structured. Ultimately, this study will help develop text-formatting guidelines to facilitate learning and memory for facts through distance education media.

\section{ACKNOWLEDGEMENTS}

This paper was completed while the third author was a doctoral student at Kansas State University.

\section{REFERENCES}

Advanced Distributed Learning (2004). Sharable Content Object Reference Model ( $2^{\text {nd }}$. ed $)$.

Ferstl, E. \& Kintsch, W. (1999). Learning from text: Structural knowledge assessment in the study of discourse comprehension. In H. van Oostendorp and S. Goldman (Eds.). The Construction of Mental Representations During Reading (pp. 247-277). Lawrence Erlbaum: New Jersey.

Jones, K., Elgin, P., Johnson, B., \& Farris, J. (2004). Internetbased distance education materials: Does writing style matter? Proceedings of the Human Factors and Ergonomics Society $48^{\text {th }}$ Annual Meeting, 1543-1547.

Kintsch, W. (1998). Comprehension: A paradigm for cognition. New York: Cambridge University Press.

Mayers, R. (2001). Multi-media learning. New York: Cambridge University Press.

Morkes, J., \& Nielsen, J. (1997). Concise, scannable, and objective: How to write for the web. Retrieved October 15, 2000, from http://www.useit.com/papers/webwriting/writing.htm 1

Schvaneveldt, R. (1990). Pathfinder associative networks: Studies in knowledge organization. Norwood, NJ: Ablex Publishing.

Shapiro, A. (1999). The relevance of hierarchies to learning biology from hypertext. The Journal of the Learning Sciences, 8(2), 215-243.

Zwaan, R. (2004). The immersed experiencer: Toward an embodied theory of language comprehension. In B. Ross (Ed.) The Psychology of Learning and Motivation, Vol. 44 (pp. 35-62). New York: Academic Press. 